\title{
Caesarean Scar Endometriosis
}

Nesuma Sedhain, ${ }^{1}$ Ganesh Dangal, ${ }^{1}$ Aruna Karki, ${ }^{1}$ Hema K Pradhan, ${ }^{1}$ Ranjana Shrestha, ${ }^{1}$ Kabin Bhattachan, ${ }^{1}$ Rekha Poudel, ${ }^{1}$ Nishma Bajracharya ${ }^{1}$

${ }^{1}$ Department of Obstetrics and Gynecology, Kathmandu Model Hospital, Kathmandu Nepal.

\section{ABSTRACT}

Endometriosis is a common benign gynecologic disorder defined as the presence of endometrial glands and
stroma outside of the normal location. The overall prevalence is $8-15 \%$ in women of reproductive age group. Scar
endometriosis is a rare disease, and is difficult to diagnose. It is commonly seen following obstetrical and gynecological
surgeries. The symptoms are non-specific, typically involving abdominal wall pain at the incision site at the time of
menstruation and palpable tender mass in the incision site. The diagnosis is frequently made only after excision of the
diseased tissue and its histological analysis. We present here a case of abdominal wall scar endometriosis in a woman
who had undergone a caesarean section four years ago. Surgical excision led to the diagnosis of scar endometriosis.
The pathogenesis, diagnosis and treatment of this rare condition are discussed.
Keywords: Abdominal wall; caesarean section; painful scar; scar endometriosis.

\section{INTRODUCTION}

Endometriosis is defined as presence of functional endometrial tissue (glands and stroma) in any site outside the uterus. It is commonly seen in ovaries, culde-sac, fallopian tubes, broad ligament, round ligament, uterosacral ligament, rectovaginal septum, vagina, caecum, ileum and rarely in urinary bladder, ureter, umbilicus, pericardium, pleura and previous abdominal surgical scar. ${ }^{1}$ The classic theories of the pathogenesis of endometriosis are: retrograde menstruation (tubal regurgitation), vascular or lymphatic spread, and coelomic metaplasia. ${ }^{2}$

Endometriosis in laparotomy scars most frequently occurs after operations in which the uterus has been opened. Scar endometriosis has been seen mostly after obstetrical or gynecological surgeries such as cesarean section, hysterotomy, hysterectomy, episiotomy, and tubal ligations. ${ }^{1,3}$ Studies have shown the incidence of scar endometriosis to be estimated around $0.08 \% .{ }^{4}$ Endometriosis, in surgical scars, is more common in the abdominal skin and subcutaneous tissue compared to muscle and fascia. ${ }^{2}$

We describe two cases of scar endometriosis, and reviewed the literature to illustrate signs and symptoms that may lead to an earlier diagnosis and prompt treatment.

\section{CASE 1}

A37-years, P2L2 presented to the outpatients department with complaints of cyclical pain and swelling on the left side of the transverse caesarean section scar for the last four years. She underwent two cesarean deliveries fourten and four years ago. She started menstruating after four months of her second caesarean, when she had this complaint of pain in the left side of caesarean scar. The pain started 2 to 3 days before the menses and peaked during the menstrual period and then gradually decreased after 2 to 3 days of menses and this phenomenon has been continuing since then. She had to take injectable analgesics to relieve the pain during every menstrual cycle. She described noticing a swelling above cesarean scar on left side where she had pain. She gave no history of discharge or bleeding from the pain site. She was under progestin in oral as well as in Depot form for nearly three years, her pain decreased in intensity but still was persistent and now her menstrual cycle was irregular and had inter-menstrual bleeding. She had no significant medical history.

Physical examination revealed a healed caesarean scar, with a nodular, tender mass of $3 \times 3 \mathrm{~cm}$ at the left part of the scar which was non-mobile. Transabdominal ultrasound showed a subcutaneous ill-defined hypo echoic lesion measuring $16.5 \mathrm{~mm} \times 12.9 \mathrm{~mm}$ in size noted in left side of scar in lower abdomen, likely endometriosis with normal uterus and ovaries. Based on history and physical examination findings, diagnosis of scar endometriosis was made. The patient was planned for a wide local excision of the mass. Intra-operatively, the lump was about $3 \times 3 \mathrm{~cm}$, firm in consistency, fibrosed in the sub-cuticular plane extending up to the abdominal 
wall muscles. Wide local excision was performed. Postoperative period was uneventful and she was discharged on day 5. On histopathology of excised mass showed fibro-adipose tissues with endometrial glands and stroma, confirming the diagnosis of scar endometriosis.

\section{CASE 2}

A 34-years, P2L2 lady presented to the OPD with complaints of pain and swelling on the left lower abdomen in an area of previous surgical scar for last two years. After a vaginal delivery 8 years ago, she had one cesarean delivery 2 years ago. She complained of pain and swelling in the left side of the cesarean scar every cycle during her regular menstrual period. She noticed the swelling to increase in size but no discharge or bleeding was noted from the pain site. Upon consultation Fine Needle Aspiration Cytology (FNAC) was done which revealed sheets and clusters of benign epithelial cells and macrophages in the background suggestive of endometriosis. She was treated conservatively with oral progestins for one month but symptoms did not improve. Hence, she was referred to our institution for operative management. She has a history of laparotomy (ovarian cystectomy) done for endometriosis 4 years ago.

On examination a healed caesarean scar, with a nonmobile, solid, nodular, tender mass of $4 \times 4 \mathrm{~cm}$, was noticed one inch above left part of the scar. Transabdominal ultrasound showed a well-defined, obliquely oriented, heteroechoic lesion measuring $31 \mathrm{~mm} \times 19 \mathrm{~mm}$ in size noted in the intramuscular plane of parietal wall at left paramedian location of pelvis with adjacent muscle fibers appear splitted by the lesion, likely endometriosis or inflammatory granuloma(Figure 1). Based on history and examination findings, she was diagnosed as a case of scar endometriosis and planned for a wide local excision of the abdominal wall lump.

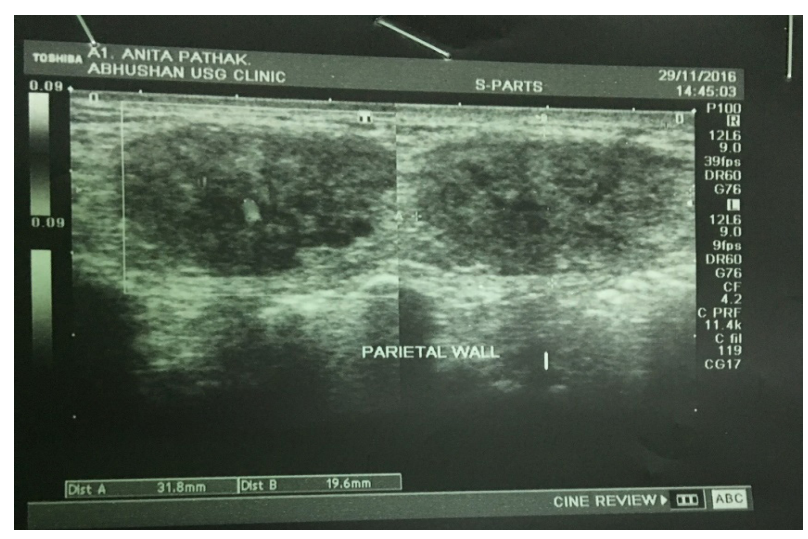

Figure 1. USG picture showing a heterogeneous hyperechoic area in parietal wall.
Intra-operatively, a $4 \mathrm{~cm}$ wide transverse incision was given $3 \mathrm{~cm}$ above the left side of previous cesarean scar. The lump was about $4 \times 4 \mathrm{~cm}$, firm, separated from rectus sheath and underlying rectus muscle and some part of peritoneum (Figure 2, 3). Wide excision was performed with electro cautery. Post-operative period was uneventful and her pain subsided. She was prescribed oral Dienogest for 1 month. Histopathology report of excised mass showed fibro-collagenous tissues with foci of normal endometrial glands and stroma embedded within the fibrous tissue (Figure 4), confirming the diagnosis of scar endometriosis.

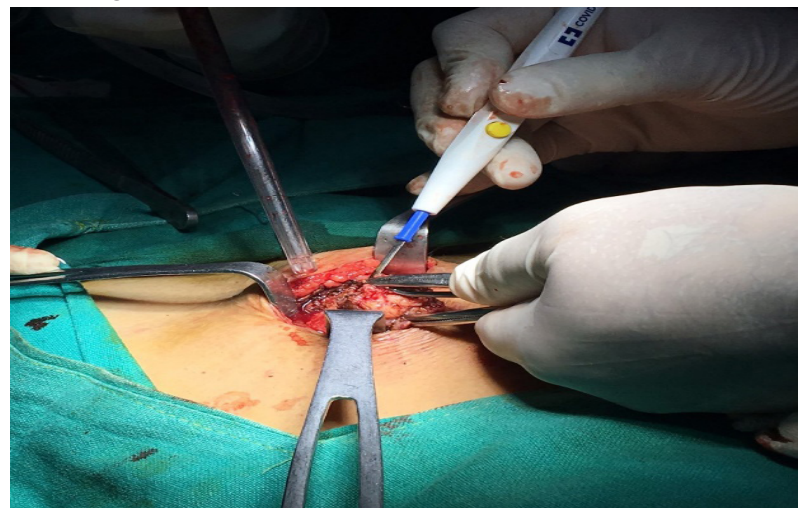

Figure 2. Gross photograph showing wide excision of abdominal mass.

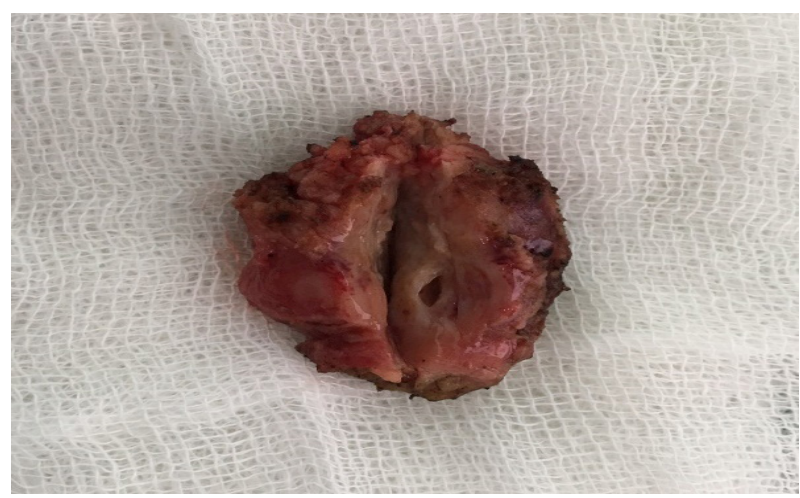

Figure 3. Gross photograph showing grey-brown fibrous area in the parietal peritoneum.

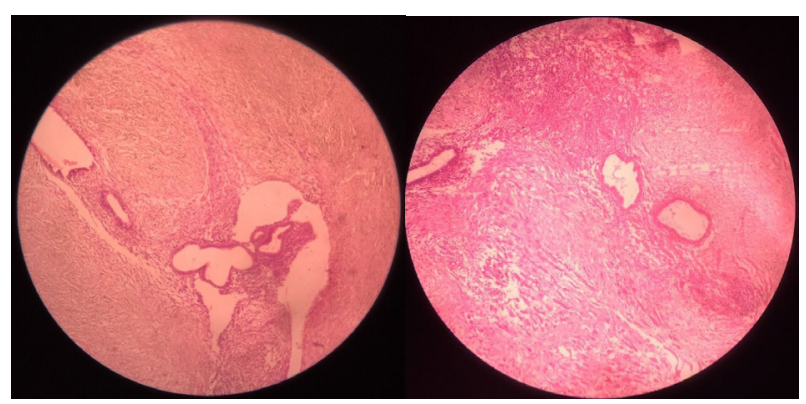

Figure 4. Histological photographs showing endometrial glands and stroma. 


\section{DISCUSSION}

Scar endometriosis, also known as incisional endometriosis refers to endometriosis occurring in surgical scars. It is called an endometrioma when a mass lesion was formed at the site. ${ }^{5}$ Meyer first documented a case of scar endometriosis in 1903 and gynecologic surgeries are the most common provoking factors of which hysterectomy (2\%) and caesarean section (< $0.4 \%)$ are the most common ones. ${ }^{5}$ Tubal ligation, laparotomy for ectopic pregnancy, salpingectomy and episiotomy, and others are the uncommon causes. ${ }^{6}$ They usually present with a painful nodule in a woman with previous history of gynecological or obstetrical surgery. The intensity of pain and size of nodule vary with menstrual cycle. ${ }^{7}$ Non-invasive imaging modalities like USG with color Doppler, CT scan and MRI scan can be highly suggestive but not diagnostic. FNAC is a valuable diagnostic tool. ${ }^{5,6}$ Cytosmears show sheets of epithelial cells, spindled stromal cells and a variable number of hemosiderin laden macrophages. Presence of two of the above findings is diagnostic. ${ }^{5}$ The cytological features can vary with cyclical hormonal changes. In the proliferative period epithelial cells form cohesive sheets with scant cytoplasm, round/oval bland nuclei. In the secretory, phase can have slight increase in size of nucleus and cytoplasmic micro-vacuolation. Uncommon cytological findings reported include squamous, tubal and mucinous metaplasia and even malignant transformation. ${ }^{5}$ Differential diagnoses vary depending on the location and the common ones are desmoid tumor, fibrosis, suture granuloma, nodular fasciitis, adnexal tumor and others. ${ }^{1}$ The treatment of choice is always wide local excision of the lesion, which serves both diagnostic and therapeutic benefit at the same time. ${ }^{6}$ Medical treatment frequently used such as progestogen, oral contraceptive pills, and danazol is not effective. They only give partial relief in symptoms however doesn't resolve the lesion.

\section{CONCLUSIONS}

Endometriosis in surgical scar tissue is a rarely seen. A high degree of clinical suspicion for endometriosis should be made, especially in women of reproductive age group, in all cases presenting with mass lesions adjacent to previous surgical scars.

\section{REFERENCE}

1. Khan AS, Inayat K, Riaz M, Nadeem S. Endometriosis at Caesarean Section Scar. Int J Pathol. 2015;13(1):41-3. [Full Text]

2. Manerikar K, Singh G, Kore R, Nongmaithem M. Abdominal wall endometriosis: a case report and review of literature. Int J Surg. 2016;3(2):995-7.[Full Text]

3. Jeyaseelan S, Kwatra N. A Rare Case of Episiotomy Scar Endometriosis. J Obstet Gynecol Ind. 2016; 66(S2):S654 S655.[Full Text]

4. Minaglia S,Mishell DR, Ballard CA. Incisional endometriomas after caesarean section: a case series. J Reprod Med. 2007;52(7):630-4.[PubMed]

5. Santosh T, Patro MK. Cytodiagnosis of scar endometriosis: A case series. J Case Rep Images Pathol. 2016;(2):29-32. [Full Text]

6. Vellido-Cotelo R, Muñoz-González JL, Oliver-Pérez MR, Hera-Lazaro CDL, Almansa-Gonzalez C, Perez-Sagaseta C et al. Endometriosis node in gynaecologic scars: a study of 17 patients and the diagnostic considerations in clinical experience in tertiary care center. BMC Women's Health. 2015;15:13.[Full Text]

7. Miccini M, Gregori M, Ferraro D, Ciardi A, Cassibba S, Biacchi D. Abdominal scar endometriosis: case report. Clin Exp Obstet Gynecol. 2016;43(3):431-3.[PubMed] 